\title{
La « Marche sur Lyon » ou le conte des deux forts
}

\section{Gregory LEE}

\section{(2) OpenEdition \\ Journals}

Édition électronique

URL : http://journals.openedition.org/transtexts/514

DOI : $10.4000 /$ transtexts. 514

ISSN : 2105-2549

Éditeur

Gregory B. Lee

Référence électronique

Gregory LEE, «La « Marche sur Lyon » ou le conte des deux forts », Transtext(e)s Transcultures 跨文本 跨文化 [En ligne], 9 | 2014, mis en ligne le 26 octobre 2015, consulté le 19 avril 2019. URL : http:// journals.openedition.org/transtexts/514 ; DOI : 10.4000/transtexts.514

Ce document a été généré automatiquement le 19 avril 2019.

(c) Tous droits réservés 


\title{
La « Marche sur Lyon » ou le conte des deux forts
}

\author{
Gregory LEE
}

1 En 1921 à Lyon, l'Institut franco-chinois, partie constituante de l'Université de Lyon s'apprêtait à ouvrir ses portes à une jeune élite chinoise en train de se former dans les grandes universités chinoises. Pourtant, des étudiants chinois, et en particulier des é tudiants du programme travail-études, étaient déjà implantés partout en France. Au début des années 1920 La situation économique de ces étudiants -ouvriers qui finançaient leurs cours du soir en travaillant pendant la journée, était devenue précaire. Lorsqu'ils apprirent la nouvelle de l'ouverture de l'Institut, une «délégation » représentant ces deux mille étudiants chinois éparpillés un peu partout en France dans des collèges, des lycées et d'autres écoles se retrouva à Lyon pour demander leur intégration au sein du nouvel institut. Au bout de quelques semaines, ces étudiants furent expulsés de France. Par la suite, cet événement serait appelé « la Marche sur Lyon ».

2 L'histoire de l'Institut franco-chinois (1921-1946) semble à première vue être une simple micro-histoire, un sous-chapitre de l'histoire d'une Chine qui cherchait à se fortifier et à s'équiper afin de faire face au monde industriel moderne. Mais presque un siècle après son inauguration, il est évident que les deux factions qui se trouvent face à face dans cette micro-histoire firent partie de manière plus ou moins inconsciente d'une même mouvance. L'objectif de cette modernisation (qui s'appelait simplement Occidentalisation, ou Xifanghua 西方化, pendant une grande partie du vingtième siècle) était, pour les mouvements progressistes, de renforcer la Chine, d'effacer l'humiliation de sa soumission aux puissances occidentales et japonaise et, plus tard, pour la gauche communiste, de se battre contre le capitalisme. Cependant, la clef pour la plupart des intellectuels et dirigeants chinois était la technologie; une minorité souhaitait également faire renaitre la vie culturelle et intellectuelle chinoise en adoptant des modèles occidentaux. Dans les deux cas ce qui s'opérait était une colonisation de l'esprit, qui fut en grande partie une auto-colonisation dans un processus d'intériorisation d'une idéologie qui privilégiait d'abord la science, puis, la technologie. L'imaginaire collectif 
dominant de l'élite acceptait la nécessité de suivre la voie technologique et l'accepte toujours. Le Parti Communiste, même sous Mao Zedong 毛睪東, ne rejetterait jamais cette démarche; même si, pendant un temps, la «bande des quatre » fut hostile à la technique et contre le productivisme et l'économisme, la faction qui représentait la tendance opposée (Zhou Enlai 周恩來, Liu Shaoqi 劉少奇 et Deng Xiaoping 鄧小平) ne cessa de promouvoir la logique de la croissance économique et de l'industrialisation.

Xiaoping dont le nom est souvent associe avec l'histoire de l'Institut franco-chinois fut actif dans le mouvement des étudiant-ouvriers en France avant son ouverture, même s'il n'a pas pas participé à la célèbre marche sur Lyon. Il était déjà acquis à l'idée du développement de la Chine. La querelle de la "Marche sur Lyon » peut se comprendre comme celle d'une élite des forces progressistes officielles, dont la mission était d'acquérir des connaissances techniques pour faire avancer la Chine, face à des étudiantsouvriers, que ces mêmes forces avaient encouragés et partageant eux aussi la vision d'une Chine modernisée. ${ }^{1}$

4 L'expulsion des Chinois de 1921 ne fut pas un simple cas de déportation d'étrangers indésirables. La situation était compliquée par des questions de classe sociale, de relations internationales, de politique, de militantisme du mouvement communiste émergent et sans doute aussi d'espionnage. Ces jeunes Chinois étaient d'abord les bénéficiaires, puis les victimes d'une politique de ce que de nos jours nous appelons le «soft power ». Une politique qui cherchait à établir, par le biais de son influence culturelle et pédagogique, un climat favorable à la France en Chine.

5 La mise-en-place de l'Institut franco-chinois convenait aux autorités françaises, mais également aux autorités chinoises. L'encadrement sur un seul lieu, dans un seul espace bien réglementé, d'une élite chinoise qui allait par la suite porter le drapeau d'une science française trempée d'idéologie française correspondait bien à la stratégie d'influence culturelle de la France. L'institut refusa d'accueillir les étudiants-ouvriers sans ressources, déjà éparpillés un peu partout en France et qui étaient devenus gênant pour le pouvoir chinois et pour la France. Mais avant d'examiner les débuts controversés de l'Institut lors des évènements de 1922, revenons tout d'abord sur l'histoire du corps estudiantin chinois en France.

$6 \quad$ Parmi les premiers étudiants chinois à venir en France pour y suivre des études financées par l'état furent Li Shizeng 李石曾 qui, par la suite, devait se distinguer par ses efforts en faveur des étudiants chinois et ses activités politiques, et Zhang Jingjiang 张静江 qui finança les actions du premier. Ils arrivèrent en 1901. Li devint rapidement un intime de la famille du géographe anarchiste Élisée Reclus. En 1906, tous deux furent rejoints par Wu Zhihui 吴稚晖 qui jusqu'alors était établi à Londres et portait également le nom anglicisé de Wood. Li gagna Wu à la cause anarchiste. Plus tard, Li et Wu penseraient et militeraient en faveur de l'Institut franco-chinois, mais en 1906 leur cause était l'anarchisme, ce qui à l'époque n'était pas incompatible avec leur adhésion au Tongmenghui 同盟會, ou Alliance révolutionnaire, de Sun Yat-sen. Ils établirent à Paris l'organisme anarchiste la Société du nouveau monde Xin shijie she 新世界社. En 1907, ils lancèrent la revue Xin shiji 新世纪 (Nouvelle ère) sous-titrée en espéranto La Tempoj Novaj. ${ }^{2}$ En 1909, avec le soutien financier de Zhang Jingjiang, Li Shizeng ouvrit une usine de production de tofu qui employait une trentaine de ses compatriotes tous originaires du même district que Li dans le Hebei. Ces travailleurs seraient également le premier groupe de Chinois venus faire des études tout en travaillant pour assurer leur survie. Trouvant gé niale cette façon de rendre possible les études en France à faible coût, Li Shizeng chercha 
à formaliser et à amplifier le mouvement à partir de l'établissement de la République en 1912. Il établit l'Association pour le travail-études en France, ou Liufa jianxue hui 留法俭学 会, et en même temps ouvrit une école à Pékin pour préparer les étudiants désireux de partir en France. Au mois de juin 1912, une association et une école travail-études furent établies au Sichuan. A la fin de cette même année, les deux premières promotions de l'é cole de Pékin partirent pour la France et, en juin 1913, ce fut au tour de la troisième. Pourtant, le maréchal Yuan Shikai 袁世凯, arrivé au pouvoir en 1913, décida de fermer l'é cole de Pékin, et l'école du Sichuan, en proie au harcèlement, fut contrainte de cesser ses activités.

$7 \quad$ La Grande Guerre créa en France un besoin de main d'œuvre pour remplacer les ouvriers partis au front. Li Shizeng y voyant une occasion d'avancer le mouvement travail-études conclut un accord avec les autorités françaises; chaque usine employant des ouvriers chinois s'engageait à leur fournir des cours de français.

8 Au mois de mars 1916 une école pour étudiants chinois ouvrit ses portes à Paris. Elle était animée par Li Shizeng et Cai Yuanpei. Courant 1918, une nouvelle école offrant des cours de français avancé s'établit à Pékin pour préparer une nouvelle génération d'étudiants à poursuivre des études en France. C'est cette génération qui allait être concernée par la marche sur Lyon.

9 Le mouvement études-travail atteint son apogée entre mars 1919 et décembre 1920. Par la suite, la crise économique s'abattit sur la France et, en 1921, les étudiants chinois connurent une pénurie d'argent et se retrouvèrent au chômage. L'Association pour l'éducation sino-française, ou Huafa jiaoyu hui 華法教育会, n'avait plus les moyens de les subventionner. Arrivant en France pour gérer la crise, Cai Yuanpei, en tant que Président de l'association, décida le 16 janvier 1921 que celle-ci ne pourrait plus prendre en charge les étudiants-ouvriers, ou " élèves ouvriers » selon l'appellation préférée des autorités françaises de l'époque. Le gouvernement chinois accepta de payer leurs billets de retour en Chine à ceux qui n'avaient pas les moyens de financer leur propre voyage. Mais cette compensation ne correspondait pas aux attentes des étudiants et, le 28 février 1921, le jour de la dernière subvention de l'association aux étudiants, plus de 400 d'entre eux se pr ésentèrent à l'ambassade de Chine à Paris pour demander que le gouvernement de Pékin assure leur survie matérielle et soutienne leurs ambitions de poursuivre leurs études en France.

10 En mai 1921, un Comité sino-français fut établi afin d'aider les étudiants. Pendant un certain temps, les 800 étudiants ont reçu cinq francs par jours, l'équivalent de trois euros de nos jours. Mais au mois de septembre le comité cessa ses activités et l'assistance aux é tudiants prit fin le 15 octobre.

11 La plupart des étudiants chinois venaient en France dans le cadre de ce qui devint au cours des années un programme d'études-travail qui leur fournissait une formation de base délivrée dans des collèges, des lycées et des écoles spécialisées pour les préparer à des métiers techniques. En même temps, ils travaillaient dans des ateliers et dans des usines. Dans le meilleur des cas, on formait de la main d'œuvre spécialisée.

12 L'idée de l'Institut franco-chinois (en chinois Zhongfa daxue 中法大学 qui signifie plutôt « université sino-française ») était un tout autre concept. Son but était de former une é lite qui, avant même de venir en France, eût acquis une maîtrise de la langue française. Cette élite était destinée à l'enseignement supérieur en Chine où elle devait diffuser la science française. Formés initialement à Lyon, ces étudiants restèrent à l'Université de 
Lyon pour y préparer des diplômes nationaux, ou poursuivirent leurs études dans d'autres établissements français. Beaucoup continuèrent en thèse de doctorat. Les é tudiants-ouvriers qui vinrent en France avant l'établissement de l'Institut franco-chinois (l'IFC) n'eurent pas la possibilité de profiter de cette expérience-là. Ils connurent le monde du travail. Ils furent aussi exposés à tout ce qui y était associé, y compris le syndicalisme et la radicalisation de la classe ouvrière française. Car nous sommes en 1922, la période d'après la Grande Guerre (1914-1918), des premières années de l'Union Sovié tique et de la montée du Communisme en tant que mouvement politique mondial. Le Communisme gagnait du terrain en France non seulement chez les ouvriers et mais aussi auprès des apprentis chinois.

13 Pourtant, comme nous l'avons vu, les promoteurs du mouvement études-travail, Li Shizeng et Wu Zhihui, étaient plutôt des militants anarchistes. Ceci dit, ils étaient é galement issus d'une classe privilégiée, et leurs idées anarchistes ne les empêchèrent pas de promouvoir une école pour une nouvelle élite nationale d'universitaires et de fonctionnaires.

14 Au début du vingtième siècle, les idées des anarchistes en Chine constituaient une sorte de pensée radicale hégémonique, mais la victoire des communistes en Russie, société à dominance agraire tout comme la Chine, permit de diffuser la pensée marxiste. Plusieurs des jeunes militants parmi les étudiants-ouvriers en France furent acquis à la cause communiste. Deng Xiaoping, le futur dirigeant de la Chine Populaire, faisait lui-même partie de ceux qui militaient contre la politique discriminatoire que représentait la dé cision de privilégier l'ouverture d'une grande école pour l'élite chinoise à Lyon plutôt que de soutenir les étudiants plus modestes appartenant au mouvement études-travail. Avec quelques deux cents autres étudiants, Deng et Chen Yi 陈毅, le futur maire de Shanghai et Ministre des affaires étrangères, écrivirent le 20 mai 1921 à Cai Yuanpei pour demander que le projet d'une université chinoise à Lyon (car au départ il s'agissait bien de cela, une université de Chine et pour les Chinois) soit modifié pour en faire une école industrielle afin de résoudre le problème des étudiants-ouvriers sans ressource. La lettre resta sans $r$ éponse.

15 Deng Xiaoping ne participera pas à la " Marche sur Lyon ", mais Chen Yi fut en première ligne, et, tout comme la plupart des manifestants, il fut expulsé de France. Chen Yi n'adhéra au Parti communiste chinois qu'en 1923, mais il est fort probable qu'il était déjà radicalisé au moment de la « Marche sur Lyon ». Plusieurs années après, alors qu'il était communiste, il tenta de revenir en France pour continuer ses études. Le futur communiste et proche de Mao Zedong, Cai Hesen 蔡和森, son épouse la militante féministe Xiang Jingyu 向警予, qui promouvait la libération des femmes en tant qu'objectif en soi, et Li Lisan 李立三 (1896-1967) participèrent également à la « Marche sur Lyon »; Ils furent tous expulsés. ${ }^{3} \mathrm{Si}$ les sinologues occidentaux mettent en avant leur participation, les récits officiels chinois mettent l'emphase uniquement sur Chen Yi, futur grand dirigeant du parti.

16 En traitant de la «Marche sur Lyon », il ne s'agit donc pas de la micro-histoire d'une manifestation d'étudiants mécontents suivie de leur expulsion du territoire en bonne et due forme, mais d'un épisode d'une ou de plusieurs grandes histoires du vingtième siècle : l'histoire intellectuelle de la Chine, la lutte idéologique entre communistes, anarchistes et féministes, lesquels paradoxalement se rapprochaient de plus en plus du pouvoir nationaliste, et l'histoire des débuts des grands militants communistes. 
17 Nous allons voir que l'expulsion des étudiants-ouvriers se fit avec la complicité, ou au moins l'indifférence, des autorités chinoises. Les étudiants-ouvriers constituaient un élé ment embarrassant qui troublait la fête de la nouvelle politique de formation d'une élite chinoise moderne. Ces étudiants ne correspondaient pas au profil social désiré, et de surcroît ils constituaient des éléments politisés à contre-courant du positionnement de la direction du nouvel institut qui tenait à exclure de manière très stricte toute activité politique. Or, avec la prétendue "Marche sur Lyon», qui fut plut ôt une tentative d'occupation, les organisateurs démontrèrent qu'ils étaient pleinement engagés dans des activités politiques spectaculaires.

La volonté de créer un établissement élitiste est clairement exprimée dans une « Note sur l'institut franco-chinois » rédigée fin 1921 après les évènements du mois de septembre, où l'on peut lire :

Pour la première promotion, il a été convenu que la sélection des étudiants aptes à entrer à l'Institut de Lyon ; École normale pour les Chinois, se ferait d'une part en Chine ; d'autre part en France ... ${ }^{4}$

C'est le cas aussi dans une minute rédigée par le préfet du Rhône à l'intention de la Sû reté générale le 28 août 1923 où est soulignée l'intention de former une élite à la française issue de l'élite existante en Chine :

L'Institut franco-chinois de Lyon est en quelque sorte une École normale supérieure [souligné dans le texte] où de jeunes Chinois et de jeunes Chinoises titulaires de certains diplômes universitaires chinois ou désignés après concours viennent compléter leurs études et s'imprégner de la culture française.... Il relève du ministère de l'Instruction publique et il est placé sous l'autorité de M. le recteur de l'Université de Lyon.

Il y avait alors deux voies de sélection: par concours (compétence en langues française, anglaise et chinoise, et en mathématiques); et via une sélection sur dossier d'étudiants diplômés d'une liste restreinte d'établissements « d'excellence » en Chine mais également au Japon où jusqu'alors un grand nombre de Chinois étaient allés chercher une éducation occidentale. Les établissements concernés étaient les suivants :

Université nationale de Pékin ;

École nationale de Médecine de Pékin ;

Écoles normales supérieures de Canton, Wuchang, etc.

École technique de Tangshan dépendant du Ministère des communications,

École Navale de Canton;

École de Médecine Paul Doumer à Canton (française);

École française du Sacré cœur à Canton (Frères Maristes) ;

Ancienne école allemande de Shanghai (rouverte en 1921 comme École franco-chinoise) ;

Faculté d'agriculture de Komaba (Université impériale de Tokyo) ;

Université privée de Waseda (Tokyo) ;

Université agricole de Nankin (américaine)

St John's University, Shanghai (américaine)

Le 21 septembre 1921, une centaine d'étudiants-ouvriers qui avaient convergé sur Lyon arrivèrent à l'Institut franco-chinois devant le Fort Saint Irénée. Ne parvenant pas à péné 
trer dans les dortoirs ils s'installèrent devant l'entrée. Les étudiants refusèrent de quitter les lieux; la police les chassa de force et les «transporta» au Fort de Montluc, où ils furent effectivement emprisonnés.

Il existe plusieurs comptes-rendus officiels des évènements de septembre et octobre 1921. Celui du 28 août 1923, rédigé deux ans après les faits dans une note établie par le préfet du Rhône, constitue un récit officiel «normal » relevant de l'histoire immédiate et établi pendant et peu après l'évacuation et l'expulsion des étudiants-ouvriers qui se rendirent à Lyon:

\section{Le Préfet du Rhône}

à Monsieur le Ministre de l'Intérieur

Sûreté Générale - Contrôle Général des Services de Police Administrative

En réponse à vos dépêches des 27 juillet et 23 août, j'ai l'honneur de vous adresser les renseignements suivants sur l'Institut franco-chinois de Lyon et sur les tendances des dirigeants de cette institution....

L'ouverture de l'Institut franco-chinois en septembre 1921 donna lieu à de sérieux incidents. L'institut devait recevoir le 24 septembre, à leur arrivée de Chine [via Marseille], les étudiants et les étudiantes déclarés admissibles que conduisait M. WU TCHI WEI (M. WOOD) [Wu Zhihui 吴稚晖], Directeur de l'Institut. Les 1500 jeunes Chinois arrivés en France en 1920 un an plus tôt pour y être élèves ouvriers et que la crise du chômage, le renchérissement du coût de la vie, le manque de ressource de leur Comité de patronage, avaient mis dans la gêne, en furent fort irrités, Groupés en "Association de post-scolaires chinois sans ressources," ces élèves ouvriers décidèrent un mouvement direct pour revendiquer l'Institut francochinois de Lyon. Sur un mot d'ordre, 106 d'entre eux quittèrent la Garenne; Fontainebleau, Château Thierry, St Germain en Laye, le Creusot, Montargis, arrivèrent à Lyon le 21 septembre; se présentèrent au Fort St Irénée siège de l'Institut et s'y installèrent avec la résolution d'y rester. Invités à rejoindre immédiatement leur résidence respective, ils s'y refusèrent obstinément en affirmant hautement leurs droits sur l'Institut. Le Secrétaire Général pour la Police dut recourir à la force pour leur faire vider les lieux et sur leur refus de reprendre le train; les fit transporter, faute d'autres logements disponibles, dans les dépendances du fort de Montluc où le gîte et la nourriture leur furent assurés. ${ }^{5}$

« Entre temps, le 24 septembre; étaient arrivés à Lyon les 125 élèves de l'Institut conduits par M. WU TCHI WEI ${ }^{6}$ Mais les autorités françaises et chinoises - l'ambassade avait envoyé M. Li Tchun, Vice-consul de Chine à Paris pour tenter de raisonner les étudiantsouvriers - craignaient une amplification du mouvement et il fut décidé de faire un exemple des manifestants:

[...L']attitude des élèves ouvriers la nécessité d'empêcher, par un exemple, le groupement des postscolaires de diriger de nouveaux contingents vers l'Institut franco-chinois de Lyon, firent hâter le rapatriement des 106 Chinois recueillis au fort de Montluc. Dans la nuit du 13 octobre, ils furent conduits en gare des Brotteaux et le 14 le matin, ils étaient embarqués à Marseille sur le 'Paul Lecat'.?

En suivant les communications de la préfecture du Rhône des mois de septembre et octobre 1921 nous pouvons établir une photographie du déroulement des évènements selon les autorités françaises. Les archives nous fournissent également plusieurs documents rédigés et émis par les étudiants-ouvriers.

Dans un rapport du $2^{\text {ème }}$ Bureau de la $4^{\text {ème }}$ Division de la préfecture du Rhône daté du 24 septembre 1921, rédigé par le Secrétaire général pour la Police et adressé au ministre de l'Intérieur ainsi qu'au ministre des Affaires Etrangères, la position des autorités chinoises à Paris est explicité : 
Aussitôt informé de l'arrivée à Lyon des ces jeunes Chinois, j'en ai avisé téléphoniquement la Direction de la Sûreté Générale et la Légation de Chine à Paris. Cette Légation m'a répondu qu'elle n'avait pas été prévenue ni n'était intervenue en aucune façon dans l'envoi de ces [106] étrangers à Lyon et n'a exprimé aucun désir à leur sujet.

Pourtant un télégramme fut envoyé par les étudiants-travailleurs ou "postscolaires » dans la matinée du 21 septembre pour avertir la légation et pour demander l'intervention des autorités chinoises :

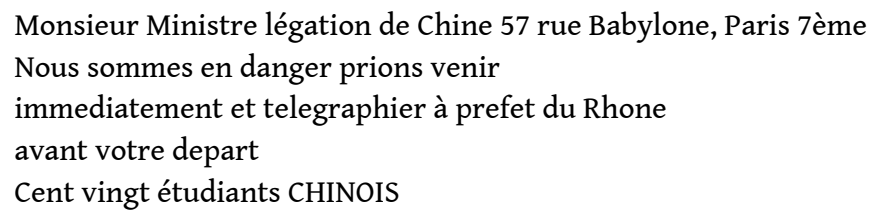

Le même jour, la Fédération des Comités des étudiants postscolaires Chinois en France émit un "Appel aux citoyens français des étudiants (sans ressource) en France, pour demander la rentrée à l'Institut Franco-Chinois de Lyon». L'appel expliquait leur situation et justifiait leurs revendications. Il stipulait que Li Yuying, secrétaire de la Société franco-chinoise d'éducation, était toujours à l'œuvre en Chine pour leur trouver une solution. De plus, les étudiants se disaient soutenus par Cai Yuanpei, président de la dite société, recteur de l'Université de Pékin et ancien ministre de l'Education. Ils citaient une déclaration du ministre de Chine à Paris du 16 septembre qui leur aurait dit :

Vous pouvez très bien entrer à l'Institut, je m'entendrai avec les Français ; car les gouvernements français et chinois subventionnent chacun une somme considérable à cet institut, ils ont donc le droit à y faire part.

41 Puis, l'appel souligne les bénéfices potentiels pour la ville de Lyon s'ils étaient admis à l'Institut :

Nous désirons bien faire de la propagande pour faciliter les relations intellectuelles et commerciales entre la ville de Lyon où nous allons faire nos études et notre pays.

Il est à désirer que les étudiants Chinois qui sont en France et sans ressources y doivent être admis d'abord.

Selon le rapport du Secrétaire général pour la Police, le vice-consul arriva le 24 septembre à Lyon. Il s'efforça « d'obtenir...l'admission de ses nationaux à l'Institut Franco-Chinois de notre Ville [Lyon] » et il poursuivit ses pourparlers « tant avec le bureau d'Administration de l'Institut qu'avec M. le maire de Lyon [Edouard Herriot] ». Estimant que les cent-six étudiants n'obtiendraient probablement pas satisfaction, le Secrétaire général pour la Police se tourna vers le gouvernement français.

Le 25 septembre, les étudiants post-scolaires émirent un "Complément » à leur appel dans lequel ils refusent de se voir représentés comme les ennemis des étudiants entrés à l'Institut franco-chinois suite aux concours passés en Chine :

Nous ne sommes pas deux fractions ennemies. Ceux qui arrivent et ceux qui sont chassés, et la place ne manque pas à l'Institut pour nous loger, si l'on s'en donne la peine, pas plus que les sources pour alimenter le budget.

Nous sommes tous des étudiants et les papiers saisis par la police en sont la preuve formelle.

Il s'en suivi une série de communications de la part des étudiants détenus, écrites sur un ton aussi désespéré que pathétique. Dans le brouillon d'une lettre datée du $1^{\mathrm{er}}$ octobre, sans destinataire, ils déclarent leur intention de faire une grève de la faim :

Mes chers Messieurs,

Nous nous permettons de porter à votre connaissance que : 


\begin{abstract}
A l'occasion de la Fête Nationale de la République de Chine; le 10 octobre, et dans la France qui réclama, la première du monde, la Liberté et le Droit de l'homme, les malheureux Etudiants Chinois postscolaires emprisonnés depuis le 22 septembre au fort Montluc de Lyon, simplement à cause de leurs revendications auprès de l'Institut Franco-Chinois de Lyon, éprouvent leur situation d'autant plus douloureuse que d'avoir obtenu peu de confiance de tous les côtés une journée de jeûne pour mieux impressionner leur inoubliable souvenir de cette fête.

Les Cent vingt étudiants Chinois postscolaires au fort Montluc
\end{abstract}

Notons que les étudiants soulignent leur exclusion du Fort St-Irénée et leur incarcération en ajoutant aux «Cent vingt étudiants Chinois postscolaires» la mention « au fort Montluc» .

Le 3 octobre, la « Fédération des étudiants postscolaires en France » écrivit au préfet. Le document que nous avons pu consulter n'est que le brouillon d'une lettre, mais nous savons que le contenu en fut lu par la préfecture car il est tamponné par le cabinet du préfet le 4 octobre $1921 .^{8}$ Les étudiants y rendent compte de la réunion qui se tint le 27 septembre entre les étudiants, Monsieur Wood, le directeur de l'Institut, et l'envoyé de l'ambassade, Monsieur Li Tchun. Pendant la réunion les étudiants auraient exposé leurs demandes comme suit :

$1^{\circ}$ L'institut prendrait autant de postscolaires que son bâtiment vacant le puisse recevoir,

$2^{\circ}$ Ceux qu'on ne peut pas recevoir ont droit d'être admis aux écoles désignées avec des conditions proportionnelles au point de vue financier,

$3^{\circ}$ Une commission de finance s'occupant des postscolaires sera constituée par l'Institut, le comité de patronage, les gouvernements Français et Chinois, la Fédération des postscolaires et les personnages voulant nous être sympathiques.

47 Puis la lettre mentionne un accord entre les étudiants et Monsieur Wood qui promit que «les conditions se réaliseraient entièrement quoi qu'un peu plus tard». Selon les étudiants, Wood se serait déjà chargé « de faire parvenir de Chine une annuité de 12 mille dollars au minimum ». Wood aurait également demandé aux représentants des étudiants de l'accompagner à l'ambassade à Paris pour avoir un entretien avec l'ambassadeur « afin d'obtenir son consentement de participer [à] la réalisation de notre demande en faisant emprunt suffisant pour nous soutenir dans un délai tout [de] six mois ». Monsieur Li Tchen leur aurait dit que la police avait autorisé par téléphone la sortie de la délégation du Fort Montluc le lendemain matin, le 28 septembre, afin qu'ils pussent emporter à Monsieur Wood la réponse de leurs camarades à Montluc.

Mais le lendemain de la réunion avec le vice-consul et Monsieur Wood, la délégation se trouva bloquée à l'intérieur de Montluc par "une défense absolue de sortir» et leur communication "avec les extérieurs...presque toute coupée». Les étudiants demandent dans leur lettre : «Pourquoi n'avons-nous point de liberté de circulation depuis le 28 [septembre] ». Les étudiants s'inquiétaient des rumeurs répandues par la presse locale :

Quelques journaux, nous savons bien, ont publié que 400 à 500 postscolaires avaient été annoncés en route sur Lyon le 28 dernier, ce que cela n'est point arrivé jusqu'à présent est assez pour prouver n'a rien de vrai.

Le Progrès a publié d'ailleurs qu'on va choisir entre nous les plus instruits et faire rapatrier les autres ineptes et indésirables....

[...] avant que nous sommes venus à Lyon, Monsieur le Ministre de Chine [l'ambassadeur] a affirmé à nos représentants, malgré son offre de rapatriement d'autre fois, que pendant sa présence en France, il protesterait fermement qu'aucun postscolaire ne serait de retour en Chine. Nous le croyons pas changé d'avis. 
Le 6 octobre une deuxième lettre, datée du 5 octobre, de la part des « cent vingt étudiants chinois post-scolaires au fort Montluc, est reçue par la préfecture dans laquelle ils demandaient leur libération. Le même jour Aristide Briand, Président du Conseil de la République, dans sa capacité de ministre des Affaires étrangères, envoya un télégramme au Maire de Lyon, Edouard Herriot :

Ne recevant pas de vous avis que Gouvernement Chinois vous facilite organisation maintient à Lyon élèves travailleurs Chinois je considère que accord intervenu entre nous sur rapatriement des cent dix élèves chinois que hospitalisés depuis quinze jours doit être exécuté je préviens messageries maritimes que les élèves seront embarqués le 13 [octobre] au soir et dirigés sur Marseille par vos soins avec la surveillance voulue dans le train indiquez-moi télégraphiquement mesures prises pour que je prévienne Préfet Marseille en vue assurer $1^{\circ}$ réception des élèves à l'arrivée du train $2^{\circ}$ conduite par ses soins à l'embarquement $\& 3^{\circ}$ mesures d'ordre pour éviter toute manifestation ou difficulté Si propositions chinoises que mentionnait votre télégramme d'avant-hier se précisent, elles pourront bénéficier aux autres élèves ouvriers chinois - BRIAND

50 Il semble que "les propositions chinoises » auxquelles réfère Briand soient celles de Cai Yuanpei et de Li Yuying que nous retrouvons dans un télégramme envoyé à Herriot le 3 octobre dans lequel les deux hommes demandaient l'obtention de « St-Jean pour installer élèves travailleurs en y organisant cours atelier internat ». Cai et Li précisèrent qu'ils avaient "espoir obtenir gouvernement [chinois] subvention annuelle 100, 000 dollars [chinois] (= 600, 000 fr. ) ». Le 7 octobre le télégramme de Cai Yuanpei et Li Yuying est annoté : « Le Maire est de l'avis de s'en maintenir au rapatriement ».

51 En dépit des protestations des étudiants et des démarches entreprises en Chine, il fut alors décidé de procéder au rapatriement des étudiants.

52 À l'approche de la date d'embarquement certains étudiants essayèrent de s'échapper. Le 12 octobre trois étudiants essayèrent de faire le mur du Fort Montluc; "ils ont été appréhendés par le gardien » et figurent sur la liste des débarqués le 14 octobre.

53 D'autres se désolidarisèrent de leurs compatriotes et tentèrent de convaincre les autorités qu'ils avaient des moyens personnels suffisants pour poursuivre leurs études en France sans l'aide des autorités chinoises ou françaises. Le 13 octobre, la veille de l'embarquement pour Marseille, trente-quatre étudiants écrivirent au préfet en lui demandant de «bien vouloir de nous permettre rester en France pour longtemps ou quelques semaines; car nous pouvons vivre et étudier par les ressources de nos familles....aussi que nous demandons pas la rentrée à l'Institut Franco-Chinois de Lyon ». Ils furent débarqués le lendemain matin.

54 Encore plus désespérée et pathétique fut la lettre d'un certain Houang Tchao-Ping qui écrivit le 13 octobre au préfet pour annoncer qu'il avait reçu de l'argent de Chine qu'il avait mis sur un compte à la Société Générale de Montargis et qu'il était en mesure de payer la pension de son Collège. Il ajouta : «si vous ne me permettez pas ce que je vous demande magnifiquement, alors je quitterai tout suite de Lyon en Belgique ou autre état, mais ne je ne veux pas retourner en Chine ». Cette dernière phrase est soulignée trois fois.

55 Le 12 octobre 1921, le Secrétaire général pour la Police requit «Au nom du peuple français » le Chef d'Escadron, Commandant la compagnie du Rhône « de commander dix gendarmes à pied (dont un gradé) pour convoyer en chemin de fer, de la gare de LyonBrotteaux à Marseille 116 individus de nationalité chinoise dirigés sur cette dernière ville en vertu d'instructions de M. le Président du Conseil, ministre des Affaires étrangères »: 
Le «transfert des Chinois du Fort Montluc à la gare des Brotteaux » fut assuré par « 4 voitures automobiles des services de Police de l'agglomération lyonnaise» qui se rendirent dans la cour du Fort le jeudi 13 octobre à 19h30. Le 14 octobre le Préfet de Lyon informa le cabinet du ministre des Affaires étrangères par dépêche télégraphique que le "Convoi groupe élèves ouvriers chinois devant être rapatriés est parti Lyon hier minuit pour Marseille sans incident ». Parmi les expulsés, celui qui allait devenir le plus célèbre de ces étudiants de «la marche sur Lyon ", était Chen Yi le futur dirigeant communiste que nous avons évoqué plus haut.

Plusieurs années plus tard, alors qu'il était déjà communiste, il tenta de revenir en France pour y continuer ses études. Dans les archives, il existe une communication de Monsieur Baudez, Consul de France à Chengdu, datée du 28 novembre 1923, qui relate que trois étudiants expulsés en 1921, dont Chen Yi, souhaitaient « retourner en France où ils désirent reprendre le cours de leurs études. La lettre fut transférée le 18 février 1924 de la direction de la Sûreté générale du ministère de l'Intérieur au préfet du Rhône "pour avis ». Nous ne savons pas quelle suite fut donnée à cette requête. Chen Yi ne revint pas en France. Mais, selon des sources chinoises officielles, il poursuivit ses études à l'Institut franco-chinois de Pékin où il entra en 1923, la même année où il adhéra au Parti communiste chinois. ${ }^{9}$ Par la suite, il devint l'un des principaux dirigeants du parti communiste et joua un rôle dans une marche beaucoup plus ardue que celle de Lyon, la mythique Longue Marche de 1934-1935. Proche de Zhou Enlai pendant la Révolution culturelle qui débuta en 1966, il fut critiqué mais jamais limogé. Il mourut en 1972 et, exceptionnellement, Mao Zedong se déplaça pour assister à ses funérailles. Aujourd'hui il figure au panthéon des héros de l'état communiste chinois et son passage à Lyon, que les autorités chinoises appellent 中国留学生的爱国运动, ou « mouvement patriotique des étudiants chinois en France ", fait partie de cet imaginaire de "l'histoire » moderne chinoise qui lie les grands dirigeants communistes « libéraux » à la France.

Parmi les autres expulsés devenus membres du Parti communiste chinois, Xiang Jingyu en conflit avec le Comintern en raison de ses positions féministes - fut fusillée par le Guomindang (KMT) en 1928. Son mari, Cai Hesen, correspondant de Mao, fut à son tour exécuté par les autorités nationalistes en $1931 .^{10} \mathrm{Li}$ Lisan eut un parcours plus sinueux à l'intérieur du parti communiste; Alors même qu'il était ministre du Travail sous le gouvernement communiste après 1949, il était en faveur de l'indépendance des syndicats. En décembre 1951, il fut limogé pour avoir commis «les erreurs de syndicalisme et d'économisme ». Li avait insisté pour que les syndicats, en tant qu'organismes de masse, aient des fonctions différentes de celles du parti. ${ }^{11}$

\section{NOTES}

1. Le projet de l'IFC fut porté par deux grands figures politiques de l'époque, l'un français, Édouard Herriot député du Rhône (1919-1940), Maire de Lyon (1905-1940) et plus tard premier ministre de France (1924-25) et l'autre chinois, Cai Yuanpei 蔡原配 ancien ministre de l'éducation de la République de Chine et président de l'Université de Pékin (1916-1926). 
Pour l'histoire du mouvement des étudiants-ouvriers chinois en France, je m'appuie sur des sources en plusieurs langues écrites de diverses perspectives idéologiques et temporelles dont Zhang Yunhou 张允侯, Yin Xuyi 殷叙急, Li Junchen 李峻晨, Liu Fa qingong jianxue yundong (1)留法 勤工俭学运动 (1), Shanghai, Renmin chubanshe, 1980 ; Paul Bailey, " The Chinese work-study movement in France », The China Quarterly, n 115, September 1988, p. 441-46 ; Xian Yujie 鲜于洁, Liu Fa qingong jianxue yundong shigao 留法勤工俭学运动史稿, Chengdu, Bashu shushe, 1994. En français, on peut consulter Nora Wang, Emigration et politique: Les étudiants-ouvriers chinois en France (1919-1925), Paris, Indes Savantes, 2002.

2. Pendant plusieurs décennies au début du vingtième siècle, l'histoire de l'Esperanto fut étroitement associée au mouvement anarchiste en Chine, voir Gotelind Müller-Saini et Gregor Benton, «Esperanto and Chinese Anarchism 1907-1920: The Translation from Diaspora to Homeland » in Language Problems \& Language Planning, 30, 1, 2006, pp. 45-73.

3. Paul Bailey, "The Chinese work-study movement dans France ", The China Quarterly, no. 115, September 1988, p. 459.

4. Pour les récits concernant la « Marche sur Lyon », je m'appuie principalement sur des dossiers de la Préfecture de Police conservés actuellement dans les locaux des Archives départementales du Rhône.

5. Minute $\mathrm{N}^{\circ} 2910$ expédiée par Mlle Moiroud le 28 août 1923.

6. Minute $\mathrm{N}^{\circ} 2910$.

7. Minute $\mathrm{N}^{\circ} 2910$.

8. La plupart des lettres des étudiants ont pour destinataire le préfet du Rhône, mais le fait que des brouillons de lettres se trouvent dans les archives de la préfecture démontre que les autorités avaient d'autres sources de renseignements et que la possibilité de l'existence de taupes à l'intérieur de l'organisation des étudiants ne peut être exclue. Nous savons aussi que des télégrammes envoyés par des étudiants furent interceptés en amont de leur envoi. Dans l'archive, nous trouvons la note suivante : «Le Chef de brigade de la salle des fils du CentralBarre a téléphoné à 12 heures 20 que des Chinois ont envoyé à leurs compatriotes, [d]es télégrammes .... Ces télégrammes ne seront expédiés que si la Préfectures le juge à propos. »

9. Voir le lien suivant: http://www.chinaspirit.net.cn/chenyi//spjj.htm (consulté le 10 septembre 2015)

10. Voir Andrea McElderry, "Woman Revolutionary: Xiang Jingyu » in The China Quarterly, no. 105, March 1986, pp 95-122. Pour les conseils de Cai Hesen à Mao Zedong voir Cai Hesen, «Lettre de Cai Hesen à Mao Zedong (13 août 1920)" in Extrême-Orient, Extrême-Occident, 1983, 2, 2, pp. 143-149 (à consulter en ligne : http://www.persee.fr/web/revues/home/prescript/article/ oroc_0754-5010_1983_num_2_2_1049). Il écrit dans sa conclusion : «il y a deux points sur lesquels il faut insister et se montrer ferme: c'est la dictature du prolétariat et l'aspect internationaliste ; il ne faut pas être entaché de nationalisme. »

11. Voir Timothy Brook et B. Michael Frolic, Civil Society in China, Armons, N.Y.,1997, p. 127.

\section{RÉSUMÉS}

En traitant l'épisode de "La Marche sur Lyon », ce texte nous rappelle le contexte houleux qui présida à l'ouverture de l'Institut à l'automne 1921. Les intellectuels chinois imaginèrent l'IFCL comme une alternative à la formule "travail-études » qui avait été initiée dès 1909 par Li 
Shizeng. Ce mouvement dont l'objectif était de permettre à des étudiants chinois de financer leurs études en travaillant dans les usines françaises s'était considérablement développé à la fin de la première guerre mondiale avant d'être victime de la crise économique de 1921. Abandonnés à leur sort, les étudiants chinois en France vont faire face au chômage et au manque d'argent. L'établissement de l'IFCL en septembre 1921 leur offrait l'espoir de pouvoir rester en France et de réaliser des études dans des conditions plus dignes. Mais l'IFCL n'avait pas été conçu pour eux. Il devait accueillir des étudiants solvables, préalablement sélectionnés en Chine et indifférents au militantisme politique, à la différence de leurs camarades du Mouvement travail-études. En s'appuyant sur des sources de première main, cet essai relate les incidents connus sous le nom de la « Marche sur Lyon » (Lida yundong 里大运动) du point de vue de l'appareil administratif franç ais. Il narre l'enchaînement des évènements qui ont provoqué la détention et l'expulsion hors de France d'une centaine d'étudiants chinois. 